\title{
Konjunkturelle Kurzarbeit - Was kann bei vorübergehendem Arbeitsausfall bewirkt werden?
}

Kurzarbeit gilt als bewährtes Instrument betriebsinterner Anpassung an einen konjunkturell bedingten, vorübergehenden Arbeitsausfall. Deshalb hat die Politik auf die aktuell tief greifende Wirtschaftskrise unter anderem mit Regelungen zur erleichterten Inanspruchnahme des Kurzarbeitergeldes reagiert. Damit konnte vorerst ein schneller Anstieg der Arbeitslosigkeit verhindert werden. Mittlerweile mehren sich aber kritische Stimmen. Sie befürchten, dass Kurzarbeit keine tragfähige Brücke über ein länger andauerndes wirtschaftliches Tal sei oder gar den Strukturwandel behindere. Der Beitrag diskutiert unter Berücksichtigung des aktuellen Forschungsstandes das Wirkungspotenzial der Neuregelungen zur Kurzarbeit. Er kommt bei einiger Kritik im Detail insgesamt zu einer positiven Bewertung.

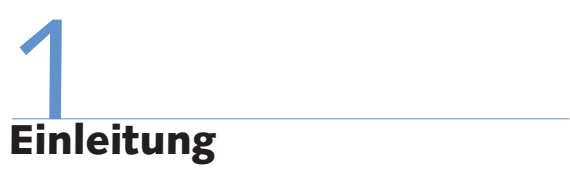

Mitte 2008 setzte der bisher in der Geschichte der Bundesrepublik Deutschlands stärkste wirtschaftliche Abschwung ein. Der erhoffte konjunkturelle Aufschwung ist derzeit nicht in Sicht. Die Wirtschafts- und Finanzpolitik sind gefordert. Zwei „Konjunkturpakte" wurden mittlerweile beschlossen. Umstritten ist, ob ein drittes Paket nützlich sein könnte. Arbeitsmarktpolitik kann hier nur unterstützen. Sie kann, wo möglich, Arbeitslosigkeit vermeiden helfen und im Blick auf den vielleicht erst 2010 einsetzenden Aufschwung einem mittelfristig absehbaren Fachkräftemangel mit der Förderung von Qualifizierungsmaßnahmen vorbeugen.

Im Mittelpunkt der aktuellen arbeitsmarktpolitischen Diskussion steht das konjunkturelle Kurzarbeitergeld (Kug) nach $\$ \$ 169 f f$. SGB III. Mit diesem klassischen Instrument der Arbeitsmarktpolitik sollen Entlassungen vermieden, d.h. bei voraussichtlich nur vorübergehendem Arbeitsund Entgeltausfall sollen bestehende sozialversicherungspflichtige Beschäftigungsverhältnisse gesichert werden. Mit vergleichbarer Zielsetzung wurde im Jahr 2006 das Saisonkurzarbeitergeld nach $₫ 175$ SGB III eingeführt. Eine andere Zielsetzung hat das Transferkurzarbeitergeld (2004 eingeführt als Modifikation des vorherigen Struktur-Kug). Es dient ausdrücklich nicht der Beschäftigungssicherung, sondern der Organisation von Übergängen in neue Beschäftigungsverhältnisse nach einem endgültigen Arbeitsausfall.
Mit den zum Jahresbeginn 2009 in Kraft getretenen und mittlerweile ausgebauten Regelungen zur Erleichterung der Inanspruchnahme des konjunkturellen Kurzarbeitergeldes wurde die Erwartung verbunden, ansonsten in großem Umfang drohende Arbeitslosigkeit vermeiden und eine tragfähige Brücke über das konjunkturelle Tal bauen zu können. In der politischen und wissenschaftlichen Diskussion werden aber auch Bedenken vorgetragen. Dies betrifft die Sorge um Mitnahmeeffekte. Vor allem aber sei bei einer länger andauernden Krise auf dem Arbeitsmarkt das Instrument der konjunkturellen Kurzarbeit überfordert, behindere möglicherweise einen erforderlichen wirtschaftsstrukturellen Wandel. Kurzarbeit verzögere dann lediglich den Eintritt in Arbeitslosigkeit und blockiere eine möglichst frühzeitige Suche nach neuer Beschäftigung (z. B. Eichhorst/Marx 2009, S. 327f.).

Kurzarbeit, insbesondere konjunkturelle Kurzarbeit, ist bis heute nur ein Randthema der Arbeitsmarktforschung. Nur wenige Studien liegen vor und können zur Einschätzung ihres Wirkungspotenzials herangezogen werden. Im Folgenden steht die konjunkturelle Kurzarbeit im Zentrum des Interesses. ${ }^{1}$ Zunächst wird der Einsatz von Kurzarbeit in der Vergangenheit betrachtet (Abschnitt 2), um die aktuelle Erleichterung der Inanspruchnahme besser einschätzen zu können. Anschließend werden die Neuregelungen kurz charakterisiert (Abschnitt 3.1), die mit ihnen verbundenen offenen Fragen aufgeworfen und es wird im Blick auf die aktuelle Umsetzung und den Stand der Forschung nach Antworten gesucht (Abschnitt 3.2). Im nächs- ten Schritt (Abschnitt 4) wird die Umsetzung der seit Anfang 2009 neuen Förderung von Qualifizierungsmaßnahmen während konjunktureller Kurzarbeit erörtert. Abschließend werden Schlussfolgerungen zum Wirkungspotenzial der Neuregelungen konjunktureller Kurzarbeit gezogen (Abschnitt 5).

\section{Inanspruchnahme von Kurzarbeitergeld in der Vergangenheit}

In den Rezessionen der 1970er und 1980er Jahre kletterte die Zahl der Kurzarbeitenden im früheren Bundesgebiet auf ein bis 2008 in Westdeutschland (mit Ausnahme

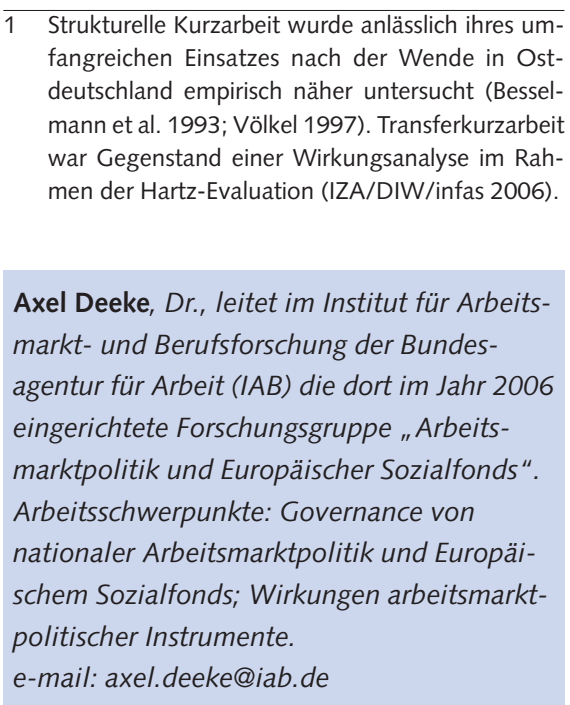




\section{Abb. 1: Kurzarbeit in Westdeutschland (ohne Berlin)}

1991 bis 2008 - Kurzarbeiter in Tsd. -

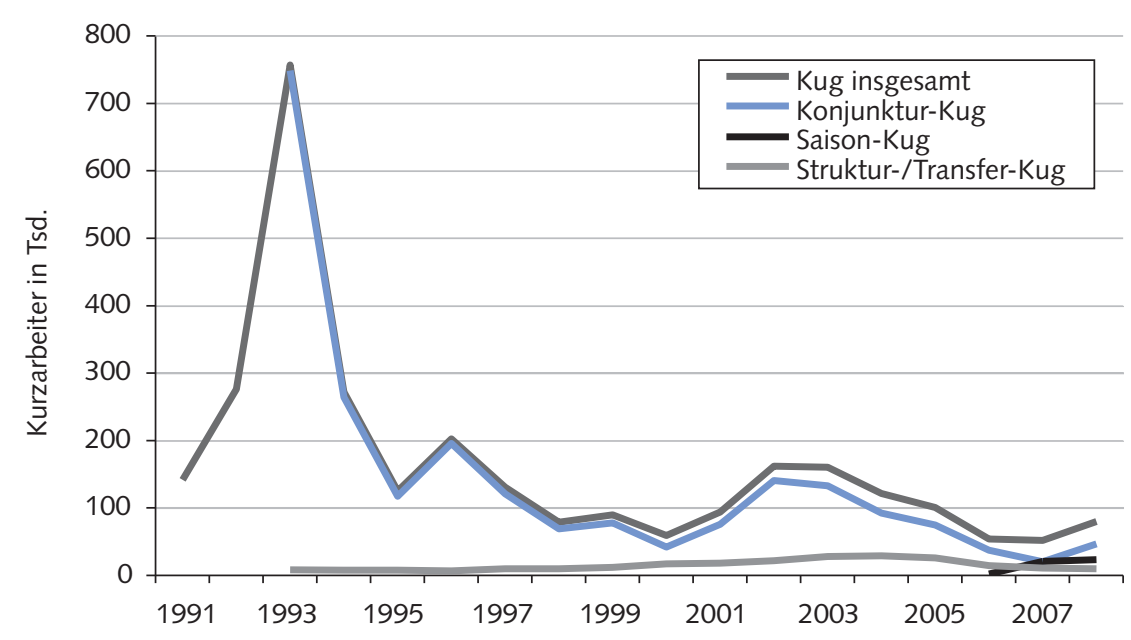

Quelle: IAB (Bach), vgl. Fußnote 2.

WSI MITTELUNGEN

Abb. 2: Kurzarbeit in Ostdeutschland (mit Berlin) 1991 bis 2008 - Kurzarbeiter in Tsd. -

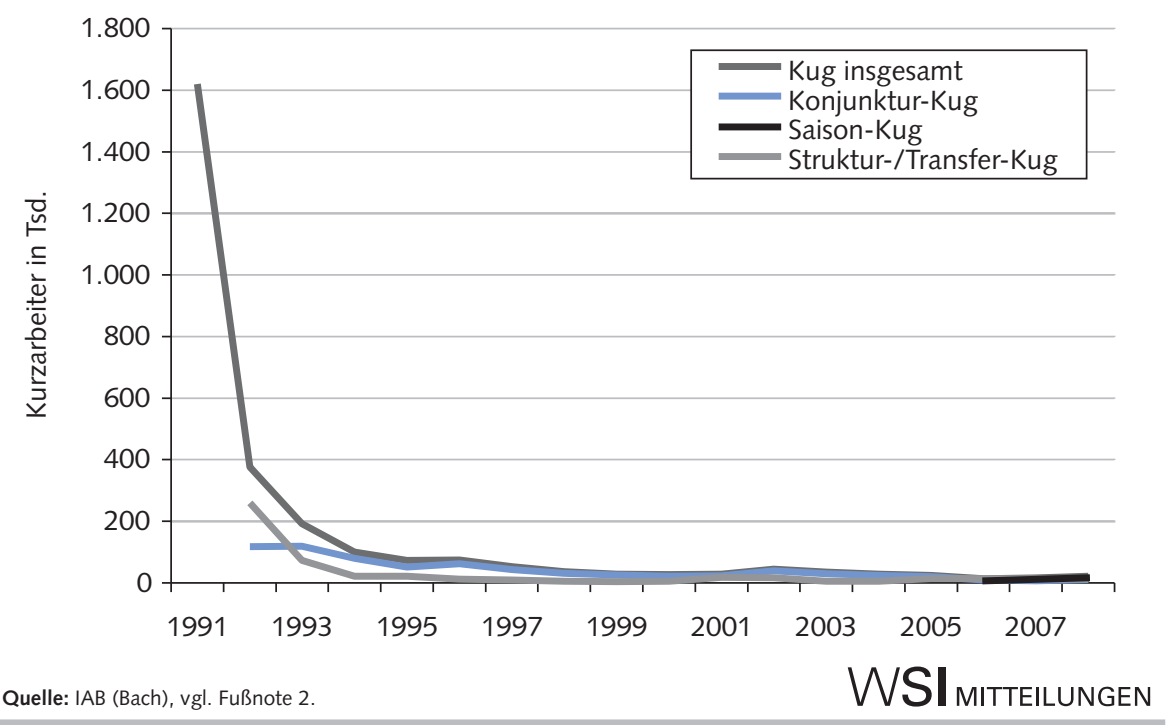

von 1993) bislang unerreichtes Niveau. ${ }^{2}$ Im jahresdurchschnittlichen Bestand von 1975 waren es 773.000 Kurzarbeitende mit einem Anteil von 3,8\% an allen sozialversicherungspflichtigen Beschäftigten, im Jahr 1983675.000 mit einem Anteil von 3,4\%. Bis Anfang der 1990er Jahre wurde dies als problematisches Wechselbad von Kurzarbeit und Überstunden diskutiert (Deeke 2005a, S. 6).

Im Jahr 1993 erhöhte sich die Anzahl der aus konjunkturellen Gründen Kurzarbeitenden im Westen noch einmal auf rund 750.000 Personen und ging dann kräftig zurück. Erst in der Rezession der Jahre 2002

uf einem relativ niedrigen Niveau (Abbildung 1).

Der Einsatz von Kurzarbeit hatte zur Zeit der Wende in Ostdeutschland eine andere Funktion als im Westen. Mit bis Ende 1991 geltenden Sonderregelungen gab es im Jahresdurchschnitt 1991 rund 1,6 Mio. Kurzarbeitende (Abbildung 2). Angesichts der zusammenbrechenden Wirtschaftsstruktur und ungewissen Zukunft handelte es sich bei diesem Einsatz von Kurzarbeit vor allem um einen sozialpolitisch inten- dierten „Fallschirm“ (Völkel 1997), der etwas modifiziert bis 1993/94 zwar auf niedrigerem Niveau, aber immer noch relativ umfänglich eingesetzt wurde. Diese strukturelle Kurzarbeit wurde in den Folgejahren weiterhin modifiziert (vgl. oben) und bis heute wie im Westen nur relativ wenig genutzt. Die hier interessierende konjunkturelle Kurzarbeit hatte dagegen im Osten von Beginn an keinen nennenswerten Stellenwert und schwankte seit der zweiten Hälfte der 1990er Jahre jahresdurchschnittlich zwischen 20.000 und 40.000 Personen. Aufgrund der ostdeutschen Sonderentwicklung wie auch der anderen Konditionen und Ziele von Kurzarbeit zur Zeit der Wende sind Schlussfolgerungen auf das Wirkungspotenzial der aktuell konjunkturell begründeten Kurzarbeit sachlich unangemessen.

Dass Kurzarbeit seit Mitte der 1990er Jahre im Westen und im Osten nur in relativ geringem Umfang eingesetzt wurde, hatte mehrere Ursachen (vgl. Deeke 2005b, S. 176ff.). Die konjunkturell bedingten Einbrüche auf dem Arbeitsmarkt waren weniger stark ausgeprägt. Der wirtschaftliche Strukturwandel weg von traditionell kurzarbeitintensiven Branchen wie der Montanindustrie trug ebenfalls dazu bei. Vor allem aber dürften die Verteuerung der Kurzarbeit für die Betriebe ab 1994 und erhöhte Anforderungen an Vorleistungen des Betriebes und der Belegschaft als Bedingung für den Bezug von Kurzarbeitergeld ausschlaggebend gewesen sein.

Seit 1994 mussten die Betriebe die Beiträge zur Sozialversicherung bezogen auf $80 \%$ des Entgeltausfalls vollständig tragen, also neben dem Arbeitgeberanteil auch den entsprechenden Arbeitnehmeranteil zahlen. Die mit der Verpflichtung zur Entgeltfortzahlung in Urlaubszeiten und Feiertagen ohnehin anfallenden Remanenzkosten wurden so nicht unerheblich erhöht. Damit wurde zugleich der Einsatz funktionaler Äquivalente interner Flexibilität für Betriebe mit absehbar vorüber-
2 Die Kurzarbeiterzahlen bis Ende der 1980er Jahre bezogen sich mit wenigen Ausnahmen nur auf konjunkturell bedingte Kurzarbeit. Strukturelle Kurzarbeit wurde erst 1988 im Arbeitsförderungsgesetz (AFG) auf eine gesetzliche Grundlage geDie hier und im Folgenden aufgeführten Zahlen zur Entwicklung der Kurzarbeit hat H.-U. Bach aus dem von ihm im IAB erarbeiteten Fundus von Langzeitreihen von Daten der BA beigesteuert. stellt und erst ab 1992/93 statistisch ausgewiesen. 
gehendem Arbeitsausfall attraktiver (Ausgleich von Schwankungen über Arbeitszeitkonten, Vereinbarungen zu befristeten Arbeitszeitverkürzungen ohne Lohnausgleich u. a.).

Zunächst „untergesetzlich“, dann mit der Einführung des SGB III im Jahr 1998 explizit im Gesetz wurde die bisherige Bestimmung präzisiert, dass der wirtschaftlich bedingte Arbeitsausfall unvermeidbar sein muss. Laut $\$ 170$ Abs. 4 ist ein Arbeitsausfall unter anderem dann nicht unvermeidbar, wenn er durch Urlaubsgewährung oder durch die Nutzung von im Betrieb zulässigen Arbeitszeitschwankungen ganz oder teilweise kompensiert werden kann. In der Regel kann also vor der Bewilligung der Kurzarbeit bzw. Zahlung von Kurzarbeitergeld verlangt werden, dass ausstehender Urlaub genommen wird, Arbeitszeitguthaben aufgelöst werden und wenn z. B. tarifvertraglich möglich - sogar eine Arbeitszeitverkürzung mit Entgeltminderung eingeführt wird. Kurzarbeit kann so vermieden oder in ihrem Umfang verringert werden (zu den weiteren gesetzlichen Regelungen vgl. Übersicht 1).

\section{Erleichterung der
Inanspruchnahme von
Kurzarbeitergeld seit
Anfang 2009}

\subsection{NEUE REGELUNGEN}

Mit Wirkung ab 1.2.2009 wurde ein neuer $\S 421 \mathrm{t}$ in das SGB III eingeführt, mit dem befristet bis Ende 2010 die Inanspruchnahme des Kurzabeitergeldes bei vorübergehendem Arbeitsausfall erleichtert werden soll. Dies wurde im Juni nochmals geändert. Zuvor wurde bereits mit Rechtsverordnung die maximale Bezugsfrist des konjunkturellen Kurzarbeitergeldes um sechs Monate auf 18 Monate verlängert und im Mai 2009 dann auf 24 Monate. Weitere Neuregelungen zielen auf eine Erleichterung der leistungsrechtlichen Verfahren zur Kurzarbeit (Übersicht 2 und 3).

Damit wurde an Stellschrauben angesetzt, die, wie oben gezeigt, seit Mitte der 1990er Jahre zur relativ geringen Inanspruchnahme des konjunkturellen Kurzarbeitergeldes beigetragen hatten. Dies betrifft vor allem die nun hälftige Erstattung der bisher allein vom Arbeitgeber zu tragen-

\section{Übersicht 1: Gesetzliche Voraussetzungen für Kurzarbeitergeld (Kug)1} bei vorübergehendem Arbeitsausfall (\$§ 169ff. SGB III)

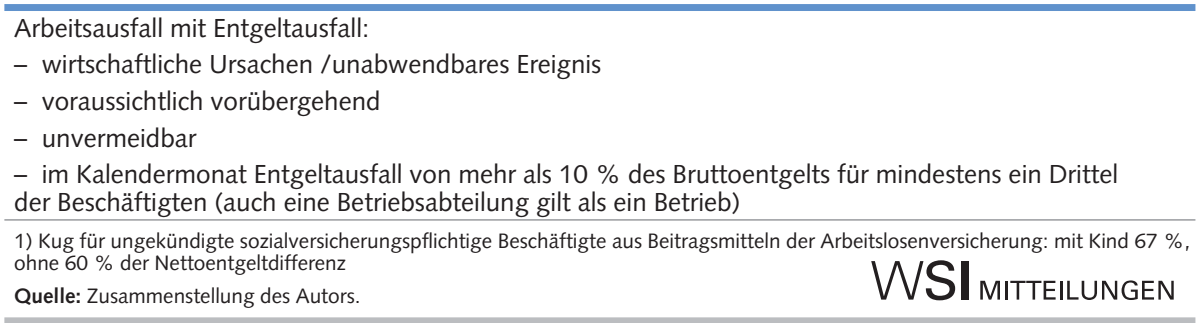

\section{Übersicht 2: Gesetzliche Neuregelung des Kurzarbeitergeldes (Kug) bei vorübergehendem Arbeitsausfall für 2009/2010}

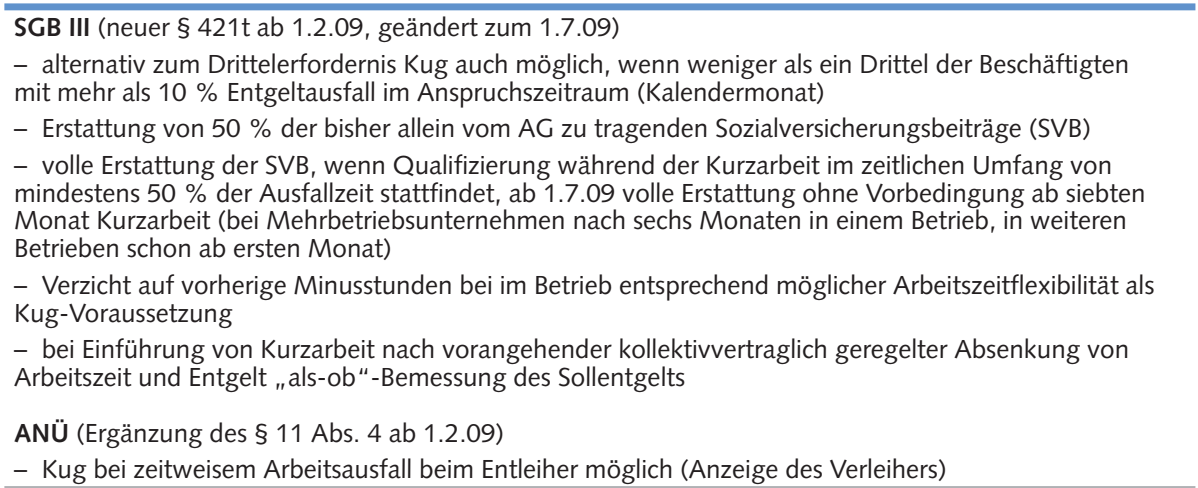

- Kug bei zeitweisem Arbeitsausfall beim Entleiher möglich (Anzeige des Verleihers)

Quelle: Zusammenstellung des Autors.

WSI MITEELUNGEN

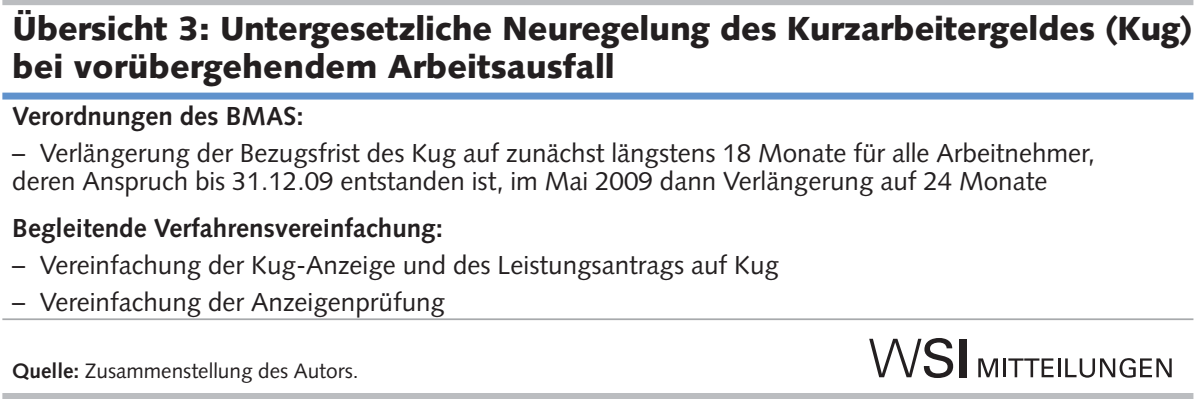

den Sozialversicherungsbeiträge und die volle Erstattung, wenn Qualifizierungsmaßnahmen für die Kurzarbeitenden durchgeführt werden. Damit sollten Anreize für die Einführung von Kurzarbeit als Alternative zu Entlassungen und zur produktiven Nutzung der Ausfallzeiten gegeben werden. Letzteres dürfte aber seit Juli 2009 wieder weitgehend entfallen, weil dann die volle Erstattung unabhängig von Qualifizierungsmaßnahmen ab dem siebten Monat möglich ist. ${ }^{3}$ Mit der zuletzt auf maximal 24 Monate verlängerten Bezugsfrist des Kurzarbeitergeldes soll den Betrieben angesichts der ungewissen wirtschaftlichen Entwicklung mehr Zeit zur Überbrückung länger andauernder Auftragseinbrüche gegeben werden.

\subsection{WIRKUNGEN}

\section{INANSPRUCHNAHME}

Zum Berichtszeitpunkt liegen die Daten zum Kurzarbeitbestand nur bis einschließlich März 2009 vor. Nach einem jahresdurchschnittlichen Bestand 2008 von 57.700 Kurzarbeitenden in 4.360 Betrieben mit konjunktureller Kurzarbeit nach $\$ 170$ SGB III stieg der Bestand bis zum März

\footnotetext{
Besonders großzügig erscheint die spezifische Subvention von Kurzarbeit in Mehrbetriebsunternehmen, derzufolge ab 1.7.09 nach sechs Monaten Kurzarbeit in einem Betrieb bei der Einführung von Kurzarbeit in weiteren Betrieben bereits von Beginn an die Sozialversicherungsbeiträge voll erstattet werden können (vgl. Übersicht 2).
} 
2009 auf dann 1,112 Mio. Kurzarbeitende in rund 36.000 Betrieben mit wirtschaftlich bedingt vorübergehendem Arbeitsausfall. Mit diesem kräftigen Anstieg der Bestandszahlen ging ein Rückgang des Arbeitsausfalls einher von im März 2008 noch 38,1 \% auf 32,5 \% im März 2009 (vorläufige Daten der Bundesagentur für Arbeit (BA)-Statistik von Ende Mai 2009).

Die BA schätzte in ihrem Monatsbericht für Mai 2009, dass auch im April und Mai dieses Jahres rund 1,1 Mio. Personen in konjunktureller Kurzarbeit waren. Das IAB rechnete in seiner im Juni 2009 aktualisierten Arbeitsmarktprojektion für 2009 weiterhin mit einer Zahl der Kurzarbeitenden in der aktuellen Höhe (Bach et al. 2009). Für das Jahr 2010 gehen die Autoren von der Annahme aus, dass „die Möglichkeiten der flexiblen Arbeitszeitgestaltung ... ausgereizt sein (dürften). Ebenso werden weniger Personen in Kurzarbeit beschäftigt werden (500.000 im Jahresdurchschnitt) zum Teil, weil es nicht mehr notwendig ist, zum Teil aber auch, weil die Unternehmen ihre Perspektiven zu pessimistisch beurteilen, um alle Kurzarbeiter wieder normal zu beschäftigen" (ebd. S. 3f.)

Die hohe Zahl der Kurzarbeitenden indiziert, dass die Wirtschaftskrise nun auf dem Arbeitsmarkt angekommen ist. $\mathrm{Zu}$ gleich scheint die Erleichterung der Inanspruchnahme von Kurzarbeit ein Erfolg zu sein, allemal dann, wenn man davon ausgeht, dass auf diese Weise in erheblichem Umfang Übergänge in offene Arbeitslosigkeit und damit Kosten von Arbeitslosigkeit vermieden werden konnten. Die Wirkungskette ist eindeutig, allerdings im quantitativen Effekt nicht genau bezifferbar.

\section{ENTLASTUNG VON ARBEITS- LOSIGKEIT}

In welchem Umfang mit Kurzarbeit von Arbeitslosigkeit entlastet wird, kann nur rechnerisch ermittelt werden. Das Institut für Arbeitsmarkt- und Berufsforschung (IAB) hat dazu bereits vor längerer Zeit ein Konzept zur Ermittlung des Beschäftigungsäquivalents der Kurzarbeit und darauf aufbauend der Entlastung der Arbeitslosenzahl entwickelt (Autorengemeinschaft 1977, S. 8). Insbesondere die Entlastungszahl wird seit einigen Jahren aus methodischen Gründen nicht mehr ausgewiesen. Bezogen auf die Zahl von 1,1 Mio. Kurzarbeitenden aus konjunkturellen Gründen im März 2009 kann mit diesem Ansatz bei dem durch- schnittlichen Arbeitsausfall von 32,5\% ein Beschäftigungsäquivalent von 362.000 Personen geschätzt werden. Wird berücksichtigt, dass ohne Kurzarbeit von diesen Personen rund $75 \%$ in offene Arbeitslosigkeit wechseln würden (der Rest in die Stille Reserve - so geschätzt in Anlehnung an den Anteil an Unterbeschäftigung 2008), kann von einer Entlastung der Arbeitslosenzahl in Höhe von ungefähr 272.000 Personen ausgegangen werden.

Das wichtigste empirische Problem dieser Schätzung besteht in der Annahme, dass es zu Entlassungen im Umfang des Beschäftigtenäquivalents gekommen wäre. Dies ist jedoch nicht sicher. Statt einer pauschalen Grobrechnung wären z. B. nach Betriebsgröße und Wirtschaftszweigen differenzierte Entlassungsrisiken in Betracht zu ziehen. Zudem können Entlassungen mit anderen Varianten betriebsinterner Flexibilität vermieden werden. Prominente Beispiele sind „betriebliche Bündnisse für Arbeit" und Beschäftigungssicherungsabkommen, die den Ergebnissen einschlägiger empirischer Studien zufolge durchaus relevante beschäftigungsstabilisierende Effekte haben können (z. B. Massa-Wirth/Seifert 2004). Ob diese früheren Befunde auch für die aktuelle Krisensituation und die damit verbundene neue Verhandlungskonstellation in den Betrieben und zwischen den Tarifpartnern gelten, ist fraglich. Aber immerhin sind nach wie vor zahlreiche tarifliche Vereinbarungen z. B. zu einer vorübergehenden Arbeitszeitverkürzung ohne Lohnausgleich in Kraft (Bispinck et al. 2009a).

Zudem besteht das Problem einer eventuellen Mitnahme des Kurzarbeitergeldes. Dieses Risiko hat Konsequenzen für die Einschätzung des Beschäftigungsäquivalents. Wenn Betriebe aufgrund der erleichterten Verfahren bei Beantragung und Bewilligung vorhandene Arbeitszeitpuffer, deren Abbau Voraussetzung für die Gewährung von Kurzarbeitergeld ist, nicht realisieren, sondern durch Kurzarbeit substituieren, ist der Umfang der Kurzarbeit, d.h. ihr Vollzeitäquivalent höher als nötig. Dies wiederum könnte die tatsächliche Entlastung von Arbeitslosigkeit durch Kurzarbeit überzeichnen.

\section{BRÜCKENFUNKTION ODER HEMMNIS IM STRUKTURWANDEL?}

Auch das Stichwort „Brückenfunktion der Kurzarbeit" verweist auf forschungsrelevante Fragen, die derzeit oft nur spekulativ gestellt und beantwortet werden. Gelingt es mit der Verlängerung der maximalen Bezugsfrist der Kurzarbeit, das Ufer des wirtschaftlichen Aufschwungs zu erreichen? Die Antwort darauf wird primär davon abhängen, ob und wann es zum Aufschwung kommt und inwieweit die Wirtschafts- und Finanzpolitik dazu beitragen. Bezogen auf Kurzarbeit geht es um zwei Fragen: Können die kurzarbeitenden Betriebe die mit Kurzarbeit generell verbundenen Kosten auch für längere Zeit tragen (z. B. bis zu 24 Monate)? Und wenn dies nicht zuletzt aufgrund der ab 1. Juli 2009 möglichen vollen Übernahme der Sozialversicherungsbeiträge seitens der BA ohne weitere Bedingungen $a b$ dem siebten Kurzarbeitsmonat in vielen Fällen machbar sein sollte - inwieweit ist dies mit strukturkonservierenden Effekten verbunden?

Mit Kurzarbeit verringern die Betriebe ihre Lohnkosten, es verbleiben aber die Remanenzkosten. Dies gilt auch, wenn sie von den Sozialversicherungsbeiträgen vollständig entlastet werden. Für arbeitsfreie Zeiten mit Entgeltanspruch fallen weiterhin Lohnkosten an, und in einigen Wirtschaftszweigen und Betrieben gibt es bei Kurzarbeit tarifliche Mindestentgeltgarantien oder eine Aufstockung zum Kurzarbeitergeld (Bispinck et al. 2009b). Dem stehen alternative Kosten für Lohnzahlungen in der Kündigungsfrist, für Entlassungen und für spätere Wiedereinstellungen gegenüber. Die Einführung von Kurzarbeit belegt, dass sie im betrieblichen Kalkül günstiger erscheint. Die Annahme, dass nicht alle Betriebe bzw. Unternehmen trotz ihrer Entlastung durch das Kurzarbeitergeld eine länger andauernde Kurzarbeit durchstehen werden, ist plausibel, kann aber nicht vorab entschieden werden. Dies sollte den Betrieben und Beschäftigten überlassen bleiben. Erst empirische Studien könnten zeigen, wie sich das betriebliche Kalkül in Abhängigkeit von der Laufzeit der Kurzarbeit und der Finanzkraft des Unternehmens einerseits und den Chancen auf eine mehr oder weniger schnelle Wiederaufnahme von Vollarbeit andererseits vor dem Hintergrund branchen- und regionalspezifischer Marktkonstellationen darstellt. Weil Kurzarbeit in der Arbeitsmarktforschung bislang wenig interessierte, fehlen aktuell entsprechende Erhebungen und Analysen zu ihrer Praxis in der Vergangenheit.

Auch mit Blick auf die Frage, ob eine Ausweitung von Kurzarbeit bis zu 24 Mo- 
nate ansonsten überzählige Produktionskapazitäten stützt, ökonomisch wünschenswerte Mobilität auf dem Arbeitsmarkt behindert und so überlebte Strukturen konserviert, gibt es empirische Forschungsdefizite. ${ }^{4}$ Mit den Statistikdaten der BA ist bisher nicht einmal beschreibbar, in welchem Umfang kurzarbeitende Betriebe die maximal mögliche Bezugsfrist des konjunkturellen Kurzarbeitergeldes nutzen. ${ }^{5}$ Abgesehen von der Möglichkeit zu entsprechenden Fallstudien dürfte der Nachweis schwierig sein, dass Kurzarbeit bei einem als vorübergehend angesehenen Arbeitsausfall den wirtschaftlichen Strukturwandel behindert.

Argumente, denen zufolge beschäftigungssichernd intendierte Kurzarbeit nicht entsprechend wirken kann, wenn ein Arbeitsplatzabbau unvermeidbar erscheint (z. B. Eichhort/Marx 2009), gehen fehl, weil sie außer Acht lassen, dass in diesen Fällen nicht von einem nur vorübergehenden Arbeitsausfall ausgegangen werden kann, die Voraussetzung für die Einführung konjunktureller Kurzarbeit also fehlt. Es könnte allenfalls Transferkurzarbeit eingeführt werden, also ein Instrument mit gänzlich anderer Zielsetzung (vgl. oben). Manche Autoren nutzen trotz heute anderer Konditionen von Kurzarbeitergeld die gleichen Argumente wie in den 1980er und 1990er Jahren, als die Unterscheidung von konjunktureller und struktureller Kurzarbeit nicht eindeutig war. Weil damals bei der Einführung von Kurzarbeit zwar gegebenenfalls für den Betrieb, aber nicht aus Sicht der Arbeitsmarktpolitik klar unterscheidbar war, ob der Arbeitsausfall voraussichtlich nur vorübergehend sein würde oder ein Arbeitsplatzabbau bevorsteht, stand das Problem im Raum. Das IAB hatte 1993 dazu Gutachten an das RheinischWestfälische Wirtschaftsinstitut (RWI) und das Kieler Weltwirtschaftsinstitut (IfW) vergeben. Darin wurde aufgrund einer Analyse der konjunkturellen Entwicklung und des Strukturwandels in den 1970er und 1980er Jahren gezeigt, dass eine eindeutige Unterscheidung zwischen konjunktureller und strukturbedingter Kurzarbeit ex-ante nicht möglich ist, weil konjunkturelle Probleme in der Regel mit empirisch letztlich nur ex-post belegbaren strukturellen Problemen verbunden sind (Klodt/Schmidt 1995; Löbbe/Siebe 1995). Dies hatte beigetragen zur ab 1998 zielorientiert am intendierten Beschäftigungseffekt der Kurzarbeit (Beschäftigungssiche- rung oder Transfer) und nicht mehr an den Ursachen des Arbeitsausfalls orientierten Neuregelung der Varianten von Kurzarbeit.

Das Argument, mit länger andauernder Kurzarbeit würde immer unsicherer, ob der Arbeitsausfall nur vorübergehend sein wird, erscheint zwar plausibel. Die Alternative dazu wäre aber der Verzicht auf die zeitliche Ausweitung der Möglichkeiten zu betriebsinterner Flexibilität mit dem Kurzarbeitergeld, also nicht selten Entlassungen. In einer Situation von Vollbeschäftigung wäre vorstellbar, dass die Entlassenen schnell wieder in Arbeit kommen. Angesichts der derzeit absehbaren Arbeitslosigkeit erscheint dies relativ unwahrscheinlich, es droht eher Langzeitarbeitslosigkeit. Nur weil grundsätzlich keine völlige Gewissheit über den Erfolg der Kurzarbeit bei vorübergehend erscheinendem Arbeitsausfall besteht, sollte nicht das alternative Risiko länger andauernder Arbeitslosigkeit eingegangen werden.

Wie bei jedem Einsatz eines Instruments der aktiven Arbeitsmarktpolitik bleibt zunächst ungewiss, ob die damit verbundenen Ziele in jedem Fall erreicht werden können. Wirkungsanalysen können bei der Einschätzung der Wahrscheinlichkeit helfen. Generell fehlen empirische Untersuchungen zu den mittelfristigen Effekten von Kurzarbeit für die Betriebe und Beschäftigten. Empirische Hinweise dazu, mit welchem Ergebnis Betriebe bei schwieriger konjunktureller Lage Kurzarbeit einsetzen, finden sich in einer Auswertung des IABBetriebspanel 2003 (Deeke 2005a).

Demnach waren im ersten Halbjahr 2003 2,3 \% aller Betriebe in Kurzarbeit, davon die Hälfte im Dienstleistungssektor und überwiegend Kleinbetriebe. Die Mehrzahl der Kurzarbeitenden fand sich dagegen im Verarbeitenden Gewerbe. 6 Die Untersuchung ergab, dass Betriebe mit Kurzarbeit ein breiteres Spektrum von Instrumenten interner und externer Flexibilität nutzten als Betriebe ohne Kurzarbeit in vergleichbarer wirtschaftlicher Situation. Ein wichtiges Ergebnis der Analyse der quantitativen Beschäftigungsentwicklung und Fluktuation im Vergleich der Betriebe mit und ohne Kurzarbeit war, dass mit der Kurzarbeit offenkundig Entlassungen vermieden wurden und die Beschäftigung stabilisiert werden konnte (ebenda, S. 19ff.).

Die Ergebnisse dieser Untersuchung stehen jedoch unter Vorbehalt. Nötig wäre eine Längsschnittuntersuchung der Be- schäftigungsentwicklung von in ihren relevanten Merkmalen möglichst ähnlichen Betrieben. Zu beantworten wäre die kontrafaktische Frage, was aus dem Betrieb bzw. seiner Beschäftigungsentwicklung geworden wäre, wenn er keine Kurzarbeit eingeführt hätte.

\section{4 \\ Qualifizierung während Kurzarbeit}

\subsection{ZWEI FÖRDERANSÄTZE BEI VORÜBERGEHENDEM ARBEITSAUSFALL}

Derzeit gibt es zwei Ansätze zur Förderung von Qualifizierungsmaßnahmen in der Zeit des Arbeitsausfalls bei konjunktureller (und saisonaler) Kurzarbeit: Erstens, im Rahmen des SGB III $\$ 77$ Abs. 2 erfolgt die volle Übernahme der Weiterbildungskosten von formal gering qualifizierten Beschäftigten in Kurzarbeit; zweitens wird in den Jahren 2009 und 2010 eine Bezuschussung der Weiterbildungskosten von Fachkräften in Kurzarbeit aus dem Europäischen Sozialfonds (ESF) im Rahmen einer Richtlinie des Bundes von Ende 2008 (ESFRichtlinie v. 18.12 .08 i. d. F. v. 10.03.09) ermöglicht. Das Kurzarbeitergeld dient je nach Umfang des Arbeits- und Entgeltausfalls mit unterschiedlichem Gewicht der Sicherung des Lebensunterhalts und im Falle der ESF-Förderung zugleich der Kofinanzierung der ESF-Mittel. Den Betrieben

\footnotetext{
4 Dies liegt nicht daran, dass lang andauernde Kurzarbeit früher nicht möglich gewesen wäre. Für die Praxis ist die gesetzlich vorgegebene Bezugsfrist von sechs Monaten seit langer Zeit nicht relevant, weil sie durch Verordnung seit den 1990er Jahren regelmäßig auf zwölf, 15 oder 18 Monate verlängert wurde.

5 Kurzarbeit kann ohne Neuantrag mehrfach für bis zu zwei Monate unterbrochen werden. Die bewilligte Bezugsfrist verlängert sich entsprechend. In der BA-Statistik wird bisher nur die ununterbrochene Dauer von Kurzarbeit ausgewiesen.

6 Nähere Angaben z. B. zur Qualifikation der Kurzarbeitenden sind nicht möglich, weil im Fragebogen dazu kein Platz war. Weil der durchschnittliche Anteil der Kurzarbeitenden an allen sozialversicherungspflichtig Beschäftigten der Betriebe mit Kurzarbeit nur bei $40 \% \mathrm{lag}$, sind Rückschlüsse aus den Angaben für die Belegschaft insgesamt nicht machbar. Deshalb kann mit den Paneldaten leider nicht gesagt werden, ob z. B. primär eingearbeitete angelernte Kräfte oder Höherqualifizierte von der Kurzarbeit profitierten (Deeke 2005a, S. 11ff.).
} 
können für die Zeit der Weiterbildung die seit Februar 2009 nur noch hälftig zu tragenden Sozialversicherungsbeiträge von der BA vollständig erstattet werden. Ab Juli 2009 soll dies nach einem halben Jahr Kurzarbeit unabhängig davon erfolgen, $o b$ ein Betrieb dann Qualifizierungsmaßnahmen durchführt. Damit wird der erst im Februar eingeführte neue Anreiz für Qualifizierung weitgehend wieder zurückgenommen.

Die Förderung der Weiterbildung von Geringqualifizierten in Kurzarbeit erfolgt mit Bildungsgutschein und setzt voraus, dass die Maßnahmen und Träger nach der Anerkennungs- und Zulassungsverordnung (AZWV) zugelassen sind. Es sollen Kenntnisse vermittelt werden, die auf dem allgemeinen Arbeitsmarkt verwertbar sind. Vorrang gegenüber der zeitlichen Lage und der Dauer der Maßnahme hat eine eventuelle Erhöhung der Arbeitszeit oder Rückkehr zur Vollarbeitszeit. Beides soll nicht durch die Teilnahme behindert werden. Allerdings ist in den Fällen, in denen es nach der Wiederaufnahme der Vollarbeit aus Sicht des Betriebes und des Beschäftigten wünschenswert erscheint, eine Fortsetzung möglich, wobei dann die Sicherung des Lebensunterhalts mit dem Arbeitsentgeltzuschuss nach $\$ 235$ c SGB III erfolgen kann.

Weil mit dem ESF nur an nationale Förderlücken angeknüpft werden kann, gilt die ESF-Richtlinie nur für Fachkräfte (nur bei im Sinne der AZWV nicht zugelassenen betriebsbezogenen Maßnahmen auch für Geringqualifizierte). Gefördert werden kann die Teilnahme an „allgemeinen Maßnahmen", in denen Qualifikationen vermittelt werden, die auf andere Unternehmen oder Arbeitsbereiche übertragen werden können. Aber auch „spezifische Maßnahmen“ kommen in Betracht, bei denen Qualifikationen vermittelt werden, die nicht oder nur begrenzt übertragbar sind. Im März 2009 wurde mit einer Änderung der Richtlinien klar gestellt, dass zwar auch Maßnahmen im eigenen Betrieb mit eigenem Personal gefördert werden können, eine Förderung aber ausgeschlossen ist, wenn es sich um Maßnahmen handelt, zu denen der Arbeitgeber gesetzlich verpflichtet ist oder die ohnehin durchgeführt worden wären. Je nachdem, ob es sich um eine allgemeine oder spezifische Maßnahme handelt sowie in Abhängigkeit von der Betriebsgröße und Personengruppen können zwischen $25 \%$ und $80 \%$ der Weiterbildungskosten erstattet werden.

\subsection{UMSETZUNGSPROBLEME}

Die Diskussion über eine Nutzung der Ausfallzeiten bei Kurzarbeit für Qualifizierungsmaßnahmen wird seit langer Zeit geführt, im Falle von konjunktureller Kurzarbeit bis Ende 2008 ohne nennenswerte praktische Relevanz. ${ }^{7}$ Vorschläge machten bereits 1980 Schmid und Semlinger in ihrer damaligen Untersuchung der Umsetzung von Kurzarbeit aus strukturellen und aus konjunkturellen Gründen (Schmid/Semlinger 1980, S. 198ff.). In der Folgezeit wurden jedoch nur in Ausnahmefällen, vor allem in der Montanindustrie, Qualifizierungsmaßnahmen im Rahmen strukturell begründeter Kurzarbeit durchgeführt. Erst mit den Sonderbedingungen struktureller Kurzarbeit in Ostdeutschland wurden Kurzarbeitende in größerer Zahl in Qualifizierungsmaßnahmen einbezogen. Dies führte zu einer Diskussion über die Möglichkeiten und Grenzen von Qualifizierungsmaßnahmen auch bei konjunktureller Kurzarbeit, die Ende 1993 von der BA in einem Erlass aufgegriffen wurde. Qualifizierungsmaßnahmen wurden darin zwar im Grundsatz befürwortet, aber in der Anpassung an Lage und Umfang der Ausfallzeiten relativ restriktiven Bedingungen unterworfen. Eine öffentliche Förderung war nicht vorgesehen. In der Praxis konjunktureller Kurzarbeit wurde die Empfehlung der BA kaum aufgegriffen (vgl. Deeke/ Schuler 2003, S. 64ff.).

Dass Betriebe ihre Kurzarbeitenden bei vorübergehendem Arbeitsausfall ohne öffentliche Förderung relativ selten qualifizieren, bestätigte sich bei Auswertung der dazu gestellten Frage im IAB-Betriebspanel 2003. Nur $5 \%$ aller Betriebe in Kurzarbeit führten für nur $3 \%$ der Kurzarbeitenden entsprechende Maßnahmen durch (Deeke 2005a, S. 32ff.).

Trotz der 2009 neuen Anreize für Qualifizierungsmaßnahmen während Kurzarbeit bei vorübergehendem Arbeitsausfall konnte nicht erwartet werden, dass dies in den ersten Monaten ab Jahresbeginn in größerer Anzahl aufgegriffen würde. $\mathrm{Zu}$ nächst stand für die Betriebe wohl die Entlastung durch Kurzarbeit im Vordergrund. Qualifizierungsmaßnahmen erfordern einen planerischen und organisatorischen Vorlauf. Daher überrascht nicht, dass es nach den - verfahrenstechnisch bedingt im Juni noch vorläufigen Angaben der BA-Statistik vom Jahresbeginn bis Mai 2009 während konjunktureller Kurzarbeit nur zu 5.000 Eintritten in ESF-Qualifizierung und 3.400 Eintritten mit SGB-III-Bildungsgutschein gekommen war. Bemerkenswert erscheint, dass von den bisher registrierten ESF-Eintritten mehr als $60 \%$ auf Betriebe mit weniger als 250 Beschäftigten entfielen.

Aus der Forschung zu betrieblicher Weiterbildung liegen mittlerweile einige aktuelle Ergebnisse z. B. zur Beteiligung unterschiedlicher Personengruppen und zur betrieblichen Weiterbildungsbereitschaft vor (z. B. Leber/Möller 2008). Fragen zur generellen Reaktion von Betrieben auf neue Ansätze zur Förderung der Weiterbildung ihrer Beschäftigten sind bisher nur zum Teil beantwortet (Spitznagel/Lott 2007). Offen ist, welchen Effekt die generelle Vollerstattung der Sozialversicherungsbeiträge nach einem halben Jahr Kurzarbeit haben wird. Zudem muss die besondere Situation der Kurzarbeit bei nur vorübergehendem Arbeitsausfall berücksichtigt werden. Die Anforderungen an die Bereitschaft der Kurzarbeitenden, die Ausfallzeit produktiv zu nutzen, und an Betriebe und Träger zu einer flexiblen und kooperativen Realisierung modularer Weiterbildungsangebote verlangen entsprechend spezifisch angelegte Anstrengungen und Konzepte „vor Ort“. Dazu könnte einschlägige empirische Forschung beitragen. Dies betrifft auch die beim gegebenen Stand der Forschung empirisch nicht beantwortbare Frage, welchen Nutzen die Kurzarbeitenden mit Qualifizierung im Vergleich zur kontrafaktischen Nichtteilnahme für ihre aktuelle und mittelfristige Erwerbsperspektive gewinnen (können). ${ }^{8}$

\footnotetext{
7 Dagegen wurden Qualifizierungsmaßnahmen während Kurzarbeit nach endgültigem Arbeitsausfall seit Mitte der 1990er Jahre mit bundesweiten ESFProgrammen gefördert. Zur in den letzten Jahren durchaus umfänglichen Inanspruchnahme und zu den Ergebnissen vgl. Deeke/Ohlert (2009).

8 Bisher fehlten dafür wichtige Datengrundlagen. Dies betrifft vor allem die Erfassung der Beschäftigten in konjunktureller Kurzarbeit ohne geförderte Qualifizierung, die als Vergleichsgruppe für Maßnahmeteilnehmer während der Kurzarbeit, die in Förderdatenbanken registriert werden, heranzuziehen wären (vgl. zu den generellen Datenproblemen einer mikroanalytischen Evaluation von Kurzarbeit und Qualifizierung am Beispiel Transferkurzarbeit Deeke/Ohlert 2009)
} 


\section{Schlussfolgerungen}

Im Unterschied zu der Anfang der 1990er Jahre in Ostdeutschland in großem Umfang eingesetzten Kurzarbeit bei endgültigem Arbeitsausfall mit einigen darauf bezogenen Forschungsprojekten stand die konjunkturelle Kurzarbeit in Deutschland bisher nur am Rande des Interesses arbeitsmarktbezogener empirischer Forschung. Abgesehen von der in den 1970er und 1980er Jahren geführten Debatte über Wechselbäder von Kurzarbeit und Überstunden gab es kaum politische oder wissenschaftliche Kontroversen zum Nutzen der Kurzarbeit, die zu entsprechender empirischer Forschung motiviert hätten.

Wenn nur relativ wenige empirische Befunde zur Wirkungsweise und zu den Wirkungen eines arbeitsmarktpolitischen Instruments vorliegen, dann spricht dies so lange nicht gegen dessen Einsatz, wie nicht gewichtige und empirisch gut begründete
Forschungsergebnisse eine Zielverfehlung belegen. Derartige Belege gibt es für konjunkturelle Kurzarbeit nicht; es gibt sie auch nicht zur Förderung von Qualifizierung während der Ausfallzeiten. Ob Kurzarbeit als Brücke zum erhofften Ufer eines wirtschaftlichen Aufschwungs trägt, kann ex-ante weder bestimmt noch mit arbeitsmarktpolitischen Instrumenten entscheidend beeinflusst werden. Es gibt aber einige empirische Evidenz dafür, dass Kurzarbeit nicht nur aktuell von offener Arbeitslosigkeit entlastet, sondern dass sie tatsächlich eine Brückenfunktion hat, die mittelfristig betrachtet zur Stabilisierung von Beschäftigung beitragen kann und so ökonomische sowie sozialpolitische Schocks auf dem Arbeitsmarkt vermeiden hilft. Weitere Forschung könnte zur Optimierung der Umsetzung sowie zur wirkungsbezogenen Ausgestaltung konjunkturell begründeter Kurzarbeit und ihrer produktiven Nutzung mit Qualifizierungsmaßnahmen beitragen.

Die aktuellen Neuregelungen zu konjunktureller Kurzarbeit erscheinen daher angesichts der tief greifenden Wirtschaftskrise angemessen - zumal sie nur befristet gelten. Allerdings: Mitnahmeeffekte können aufgrund der erleichterten Inanspruchnahme nicht ausgeschlossen werden. Verschärfte Regelungen, um Mitnahmeeffekte zu vermeiden, würden jedoch möglicherweise den Einsatz des Instrumentes zu stark hemmen. ${ }^{9}$ Handlungsbedarf besteht bei den relativ komplizierten und mit erheblichem bürokratischen Aufwand verbundenen Förderansätzen zur Qualifizierung während konjunktureller Kurzarbeit. Eine Vereinfachung des Förderrechts sollte erwogen werden. Zudem könnte durch den Ausbau von Beratungsangeboten ,vor Ort“ und mit Qualifizierungsverbünden von Betrieben vielleicht mehr erreicht werden als bisher.

9 Zudem hat die BA Prüfungsmöglichkeiten geschaffen und Rückzahlungsforderungen sind möglich. Dies betrifft sowohl das Kurzarbeitergeld selber als auch z. B. die ESF-Qualifizierung.

\section{LITERATUR}

Autorengemeinschaft (1977): Der Arbeitsmarkt in der Bundesrepublik Deutschland 1977 (insgesamt und regional) und die Auswirkungen arbeitsmarktpolitsichre Maßnahmen, in : MittAB 1, S. 1-18

Bach, H-U./Gartner, H./Hummel, M./Klinger, S./Spitznagel, E./Zika, G. (2009): Zwischenbericht zur IAB-Arbeitsmarktprojektion 2009 und 2010 vom 10. Juni 2009, http://doku.iab.de/grauepap/2009/proj0609.pdf Besselmann, K./Mochalowski, G./Ochs, C./Seifert, H. (1993): Kurzarbeit und Qualifizierung, Forschungsbericht Sozialforschung des BMA Bd. 235, Düsseldorf, Köln, Bonn

Bispinck, R./WSI-Tarifarchiv (2009a): Tarifliche Regelungen zur befristeten Arbeitszeitverkürzung?, Elemente qualitativer Tarifpolitik Nr. 67, Düsseldorf

Bispinck, R./WSI-Tarifarchiv (2009b): Tarifliche Regelungen zur Kurzarbeit, Elemente qualitativer Tarifpolitik Nr. 66, Düsseldorf Deeke, A. (2005a): Kurzarbeit als Instrument betrieblicher Flexibilität. Ergebnisse aus dem IAB-Betriebspanel 2003, IAB-Forschungsbericht 12 Deeke, A. (2005b): Kurzarbeit, in: Allmendinger, J./Eichhorst. W./Walwei, U. (Hrsg.): IAB Handbuch Arbeitsmarkt, Frankfurt a. M., S. 175-181 Deeke, A./Ohlert, C. (2009): Qualifizierungsmaßnahmen während Kurzarbeit nach endgültigem Arbeitsausfall, IAB-Forschungsbericht 3 Deeke, A./Schuler, W. (2003): Fünf Jahre „AFG-Plus": Arbeitsförderung aus dem Europäischen Sozialfonds, BeitrAB 265, Nürnberg
Eichhorst, W./Marx, P. (2009): Kurzarbeit: Sinnvoller Konjunkturpuffer oder verlängertes Arbeitslosengeld?, in: Wirtschaftsdienst 5, S. 322-328 IZA/DIW/infas (2006): Evaluation der Maßnahmen zur Umsetzung der Vorschläge der Hartz-Kommission. Modul 1b: Förderung beruflicher Weiterbildung und Transferleistungen, Bericht Juni 2006, Bonn, Berlin Klodt, H./Schmidt, K.-D. (1995): Branchenabgrenzungen und Branchenprognosen. Überlegungen zum Struktur-Kurzarbeitergeld nach § 63 Abs. 4 AFG, in: MittAB 4, S. 544-561

Leber, U./Möller, I. (2008): Weiterbildungsbeteiligung ausgewählter Personengruppen, in: Schmollers Jahrbuch 128, S. 405-429

Löbbe, K./Siebe, T. (1995): Zur Konkretisierung des Begriffs "Schwerwiegende strukturelle Verschlechterung der Lage eines Wirtschaftszweiges“ (§ 63 Abs. 4 AFG), in: MittAB 4, S. 562-572

Massa-Wirth, H./Seifert, H. (2004): Betriebliche Bündnisse für Arbeit mit begrenzter Reichweite?, in: WSI-Mitteilungen 5, S. 246-254

Schmid. G./Semlinger, K. (1980): Instrumente gezielter Arbeitsmarktpolitik: Kurzarbeit, Einarbeitungszuschüsse, Eingliederungsbeihilfen, Schriften des WZB Bd. 31, Königstein/Ts.

Spitznagel, E./Lott, M. (2007): Wenig Betrieb auf neuen Wegen der beruflichen Weiterbildung, IAB-Kurzbericht 23/2007

Völkel, B. (1997): Kurzarbeit in den neuen Bundsländern, in: MittAB 1, S. 127-149 